\title{
Advances in Therapy for Osteoporosis
}

\author{
Robert P. Heaney, MD, Osteoporosis Research Center, Creighton University, Omaha, Nebraska
}

\begin{abstract}
The current status of detection and treatment of osteoporosis is reviewed. Despite substantial advances in the past ten years, most patients with osteoporotic fractures are still not being treated for the underlying bony cause of the fracture, and most people at risk for fracture are not being offered known protective regimens. The foundation of any therapeutic program is adequate nutrition specifically protein, calcium, phosphorus and vitamin D. Current anti-resorptive agents reduce vertebral fracture risk by $30 \%$ to $50 \%$ and teriparatide, a newly approved anabolic agent, reduces risk by up to $80+\%$. Effective treatments for chronic bony pain that occurs in some patients with spine fractures is affored by two minimally invasive procedures, kyphoplasty and vertebroplasty. Some of these chronically painful fractures represent instances of previously unrecognized non-union, and in them low-pressure vertebroplasty produces prompt and lasting relief. Fracture risk reductions with current anti-resorptive agents are at least twice as great as can be explained by drug effects on bone mass. Moreover, risk is reduced within a few months of starting therapy. These observations focus attention on bone remodeling and point to the need for improvement of biomarker technology, since it seems likely that reduction in remodeling activity underlies much of the fracture risk reduction and can therefore be used to monitor therapy.
\end{abstract}

\section{INTRODUCTION}

Whenever new treatments become available for a category of disease, there often follows an explosion, not only of additional therapies, but of diagnostic and evaluative methods as well. This is generally because what had initially seemed to be a monolithic disorder comes to be recognized as a group of related problems that require different approaches and that respond differently to various interventions. Precisely these developments have characterized the field of osteoporosis since the introduction of the first bisphosphonates for the treatment of this disorder over ten years ago. Moreover, despite many important advances and new insights, it is safe to say that the field is still evolving and has not yet reached its full maturity. This review of the advances in therapy for osteoporosis will briefly discuss the bases of osteoporotic fragility, present some urgent problems, outline currently available treatments, mention some important developments in the surgical management of certain vertebral fractures, and in closing, call attention to the increasing recognition of the importance of elevated bone remodeling in many patients with osteoporosis.

REPRINT REQUESTS:

Robert P. Heaney, MD

Creighton University

Osteoporosis Research Center

601 North 30th Street - Suite 4841

Omaha, Nebraska 68131

Telephone: 402-280-4029

Fax: $402-280-4751$

Email: rheaney@creighton.edu

\section{KEYWORDS:}

Osteoporosis; Fracture; Bisphosphonates; SERMs; Calcium; Phosphorus; Protein; Vitamin D; Anti-resorptive agents; Anabolic agents; Bone mass; Kyphoplasty; Vertebroplasty; Unstable vertebral fractures; Bone remodeling 


\section{THE BASES OF OSTEOPOROTIC FRAGILITY}

Osteoporosis was redefined in 1990 as a condition of skeletal fragility caused by decreased bone mass and by microarchitectural deterioration of bone tissue, with a consequent increase in the risk of fracture. ${ }^{1}$ This redefinition correctly placed the emphasis on fracture, the morbid event we should like to prevent (or the problem with which we must contend if our prevention has failed). It also, correctly, shifted bone mass (or density) to the status of a risk factor. Figure 1, summarizes the hierarchical relationship of several broad categories of factors important in producing fracture. As is generally recognized, bones break when they are subjected to more force than their intrinsic strength is able to sustain. Figure 1 and the above definition both emphasize that falls play an important role, and that bone architecture, bone shape and bony material properties contribute significantly to strength, as does bone mass (or density).

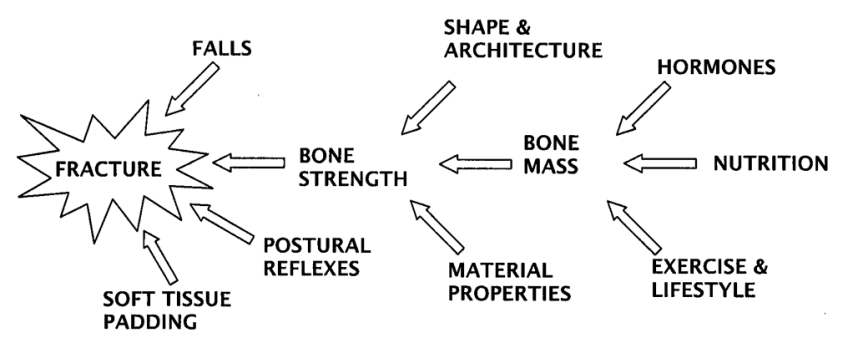

Figure 1. Hierarchical array of the major classes of factors contributing to osteoporotic fractures.

Copyright Robert P. Heaney, 1994. Used with permission.

One problem the field confronts is precisely our success in measuring bone mass with a degree of accuracy that rivals or exceeds that of tools of any other field of medicine. Thus, despite the 1990 redefinition, the working group for the World Health Organization, four years later, again classified osteoporosis in terms of degrees of bone loss. ${ }^{2}$ While there were economic and tactical reasons for this move, one consequence has been the reinforcement of the notion that osteoporotic fragility was due mainly to decreased bone density, and the ignoring of non-mass factors.

Hui et al., as long ago as 1988, dramatically showed the importance of those non-mass factors. ${ }^{3}$ These investigators evaluated the effect of both bone mass and age on fracture risk (figure 2). They observed the expected increase in fracture risk as bone density dropped, but, holding density constant, found a much greater increase in fracture risk with rise in age. While the factors responsible for this seeming paradox were not elucidated in that study, it now seems likely that changes in patterns of falling, as well as progressive, age-related changes in structural and material properties of the bone itself, are largely responsible. These and related insights have led, for example, to the development of various hip protectors, which operate by distributing the energy of a fall across a broad region of the lateral thigh, and which, when worn, produce striking reductions in fracture risk. ${ }^{4,5}$ Incidentally, these observations of the efficacy of hip protectors effectively disprove the once prevalent notion that many hip fractures occur prior to the fall and are, instead, the cause of it. In the clinical trials published to date, the hip protectors did not prevent the falls, simply the fractures. These clinical observations confirm what the mechanical engineers have long known, namely that the force of a fall from standing height, directed against the greater trochanter, is sufficient to break even a relatively strong hip. Young and middle-age individuals rarely fall in a way that exposes the upper femur to such forces, whereas elderly individuals, with little forward motion and unsteady gait, do so more often. 6

The purpose of this discussion is not to suggest that bone mass is not important. Clearly, it is: each drop of about $11 \%$ to $12 \%$ in bone density approximately doubles fracture risk. Nevertheless, structural features such as connectivity in trabecular bone and excessive trabecular remodeling are now recognized to be critically important as well. The clinical problem is that good tools for assessing their presence in patients with osteoporosis are currently not available, leading to the emphasis being placed on what can be measured. However, the undoubted importance of remodeling activity, discussed in detail below, makes it likely that better approaches to the assessment of the remodeling rate will become available soon.

\section{URGENT PROBLEMS}

There still is a segment of the medical community that discounts the importance of osteoporosis. For example, the April 13, 2002 issue of British Medical Journal contains a number of papers accusing practitioners of over-diagnosis and over-treatment, both arising out of a purported unholy alliance between the pharmaceutical industry and uncritical physician investigators. These papers ${ }^{7-9}$ specifically cited the treatment of low bone density as an instance of such inappropriate medicine. Despite such carping, it remains true that osteoporosis is a serious disorder, the full import of which is only recently becoming clear. ${ }^{10}$ For example, mor-

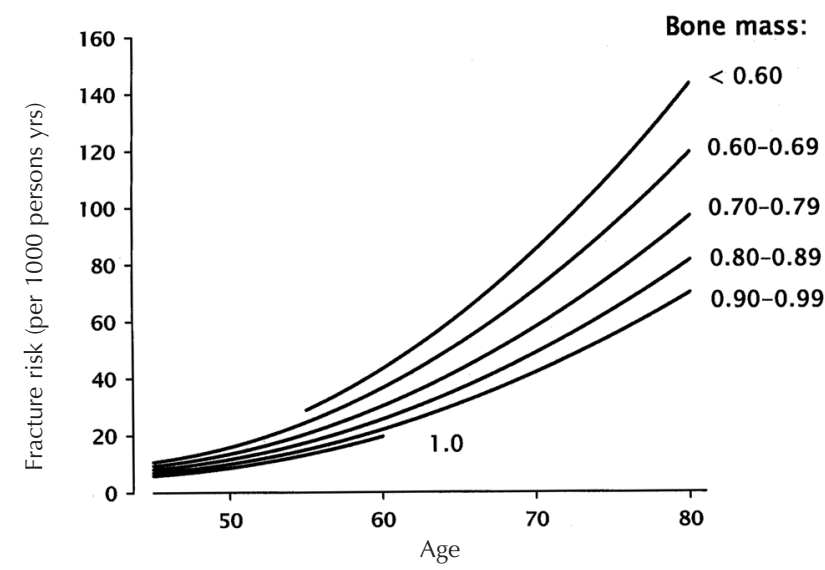

Figure 2. Gradients of fracture risk on age and forearm bone mass, expressed as BMD $\left(\mathrm{g} / \mathrm{cm}^{2}\right)$ (redrawn from Hui et al.). ${ }^{3}$ Note that, while risk rises as BMD falls, it increases even more strikingly with age, holding BMD constant.

Copyright Robert P. Heaney, 1994. Used with permission. 
tality within 90 days of most osteoporotic fractures in individuals over age sixty-five is substantially higher than would be predicted for the age concerned, and for some fractures, risk of early death rises by nearly seven-fold. Vertebral fractures, the most common of the osteoporotic fractures, themselves have significant excess mortality-both short- and long-term-in addition to their substantial impact on quality of life. Therefore, osteoporosis is worth detecting, preventing and treating.

Two particularly urgent problems can be identified. The first is that in most patients who suffer fractures now recognized to be osteoporotic, the underlying disease-osteoporosis-is generally not diagnosed or treated. Second, patients with findings that are now known to put them at risk for osteoporotic fractures are not being detected or treated in a way that would prevent first fractures. The importance of first fracture, particularly of the spine, is that prevalent fractures are highly predictive of further fractures. Various studies have estimated that the risk rises by as much as five-fold. What is not clear at this time is whether the first fracture itself predisposes to further fractures, or is simply a marker for the fragility that will inevitably express itself as further fractures. If the former, then a cost-effective intervention prior to fracture should be found. However, if the latter is the case, then at very least, those who have already sustained a fracture must be treated.

Despite the effectiveness of available agents in reducing fracture risk in patients with prevalent fracture, there is a sense in which such treatment, while necessary and appropriate, is too late. The reduction in risk that can be produced by treatment yields a treated fracture rate that is still 2 to 3 times higher than the untreated risk in the placebo groups of these same studies for individuals who had not had a fracture prior to entry. Thus, optimal use of anti-resorptive treatment agents (i.e., the bisphosphonates, Selective Estrogen Receptor Modulators [SERMs], estrogen and calcitonin) leaves patients who already had sustained one or more fractures at higher risk of further fracture, than untreated patients who have not yet sustained a fracture.

The goal of detecting and treating those at risk is still far from being achieved. The published results of the National Osteoporosis Risk Assessment (NORA) study ${ }^{11}$ made it clear that most patients with osteoporosis in primary care practices in North America were neither being diagnosed nor treated, even those with prevalent fractures. Even in teaching hospitals, the record has not been good. Gardner et al. ${ }^{12}$ summarized the experience at the Hospital for Special Surgery in New York City, shown in table 1. In 1997, only $11 \%$ of patients with hip fracture were discharged with any form of anti-osteoporosis medical therapy, even calcium or vitamin D. While this performance had improved substantially by three years later, it remained sadly true that, even then, barely one patient in four received any form of treatment that might be considered related to the underlying disease that was at least partly responsible for the hip fracture.
An explanation emerging from focus group discussions is that orthopedic surgeons are uncomfortable with long-term, outpatient management of chronic disorders. Instead, they see themselves as acute care physicians, and assume that the patient's primary care practitioner will handle the patient's medical problems and the treatment thereof. But often the medical problem is left untreated when the patient is discharged after successful surgical repair of fracture. The development of new intravenous bisphosphonates ${ }^{13}$ may provide a tactical solution to this problem. A single dose given while the patient is in hospital, produces an effect sufficiently protracted to tide the patient over for several months until a definitive medical treatment regimen can be inaugurated.

\section{CURRENT MEDICAL THERAPY}

\section{Therapeutic Foundation}

Bone substance is made out of protein and mineral. Not surprisingly, therefore, the foundation of any preventive or therapeutic regimen is an adequate dietary intake of these bulk materials: high quality protein, calcium and phosphorus. The various anti-resorptive and anabolic agents available to date are not capable of stopping bone loss or producing bone gain if the patient is in negative nitrogen and mineral balance because of inadequate intake of these nutrients. The best current estimates of optimal protein intake in the elderly are in the range of $1 \mathrm{~g} / \mathrm{kg}$ body weight; calcium intakes should be in the range of $1500 \mathrm{mg} / \mathrm{d}$; and phosphorus intakes should be at least at the level of the RDA (700 $\mathrm{mg} / \mathrm{d})^{14}$ - and possibly more if the calcium intake comes predominantly from carbonate or citrate-based supplements (since both will bind food phosphorus in the intestinal tract and reduce its availability for bone building). ${ }^{15}$

Vitamin D is also essential for bone health and operates in many body systems in addition to facilitating the absorption of ingested calcium. The recommended intake of vitamin D is $200 \mathrm{IU}(5 \mathrm{mg})$ up to age fifty, $400 \mathrm{IU}(10 \mathrm{mg})$ from age fifty to seventy, and $600 \mathrm{IU}(15 \mathrm{mg})$ above age seventy. ${ }^{14}$ The rise in recommendation with age represents not so much an increase in requirement, as a decline in cutaneous vitamin D input, because of both decreased efficiency of conversion of 7-dehydrocholesterol in the skin and decreased skin exposure with age. Despite the tripling of the recommendation with age, even 600 IU is probably inadequate for the typical elderly person. Heaney et al. ${ }^{16}$ found that it took approximately $500 \mathrm{IU} / \mathrm{d}$ to prevent serum $25(\mathrm{OH}) \mathrm{D}$ levels from falling over winter in healthy males under age fifty (or more than twice the recommended intake

Table 1. Patients discharged following hip fracture and receiving anti-osteoporosis medical therapy.

\begin{tabular}{cc}
\hline Year & Percent \\
\hline 1997 & 11 \\
1998 & 13 \\
1999 & 24 \\
2000 & 27
\end{tabular}


for that age). In elderly individuals in the osteoporosis clinic at Creighton, 1,000 IU/d year round is routinely prescribed. In no instance does this produce hypercalcemia or hypercalciuria. The importance of an adequate nutritional foundation is highlighted by the randomized controlled trials of Chapuy et al. ${ }^{17}$ and Dawson-Hughes et al., 18 both of which showed substantial reductions in hip and other extremity fractures within 18 and 36 months of starting intakes of calcium and vitamin D in the range described above. More to the point, these effects were produced without bone-active drugs. The role of protein intake has been less extensively studied.

However, it is now well established that protein supplements enhance recovery from hip fracture. ${ }^{19,20}$ In the study by Dawson-Hughes et al. ${ }^{18,21}$ bone gain with calcium supplements occurred only in those individuals with the highest protein intake.

Intakes of calcium and vitamin D recommended by the antiresorptive drug manufacturers to accompany their treatment agents tend to be substantially lower than the figures cited above. This is partly because many of the trials of these agents were designed before the importance or efficacy of calcium and the need for vitamin D were well appreciated (and hence their agents were not tested at the high calcium intakes that now seem important). In addition, brand managers are often hesitant to stress that some other conditions must be met in order for the patient to realize full benefit from the drug concerned. It is therefore incumbent upon the physician to make up for this deficiency in the promotional efforts of the pharmaceutical industry.

\section{Pharmacotherapy}

There are two broad classes of pharmacologic treatment agents now available: the anti-resorptives and the anabolics. Anti-resorptives include the bisphosphonates (alendronate, risedronate, etidronate, pamidronate and zoledronate), one selective estrogen receptor modulator (raloxifene), estrogen and calcitonin. The only anabolic agent available in the U.S. is teriparatide (recombinant human PTH 1-34). The antiresorptives generally reduce bone loss, but often they do more. All produce a remodeling transient, expressed typically as a $1 \%$ to $5 \%$ mean increase in bone mass by the end of the first year of treatment. ${ }^{22}$ All reduce the various remodeling indices that have been used to date by something on the order of $40 \%$ to $70 \%$. In general, the remodeling changes are rapidly expressed and are fully apparent within three to six months of starting treatment. Additionally, the bisphosphonates, in particular, induce a steady state, slow bone gain.

Both the bisphosphonates and teriparatide have been shown to be fully effective not only for postmenopausal osteoporosis, but for glucocorticoid-induced osteoporosis (GIO), and in both disorders, for both prevention and for treatment. ${ }^{23,24}$ Similarly, they are equally as efficacious in men as in women.

Because vertebral compression fractures in GIO, when they occur, begin so rapidly after onset of corticosteroid therapy, it is now widely recommended that a prescription for a corti- costeroid be accompanied by either a prescription for a bisphosphonate, or use of one of the intravenous bisphosphonate preparations. Even corticosteroid therapy that at the outset, is expected to be short lived, often becomes protracted, and the physician becomes alerted to the problem of osteoporosis when it is too late to prevent the first fracture. No harm would be done with short-term bisphosphonate therapy when the corticosteroid use is truly short-term, and the incremental expense of short-term bisphosphonate therapy is small. Hence this analysis strongly favors such a therapeutic approach.

\section{Anabolic Agents}

Probably the most important development in recent years in anti-osteoporosis therapy was the recent release of teriparatide. This agent is given subcutaneously, on a once-daily basis. When supported with adequate calcium and vitamin $\mathrm{D}$, teriparatide produces increases in bone mass of $10 \%$ to $15 \%$ per year. ${ }^{25,26}$ More importantly it reduces risk of all vertebral fractures by two-thirds, and of severe and multiple vertebral fractures by approximately $85 \% .^{25}$ It also reduces non-vertebral fracture rates by approximately $50 \%$. When given in combination with estrogen, teriparatide has increased spine bone density by $30 \%$, and hip bone density by $12 \%$ at two years. ${ }^{26}$ In contrast to the anti-resorptives, teriparatide acts by directly stimulating osteoblastic bone formation at both trabecular and cortical sites.

Because the agent is so new, there is virtually no practice experience to describe; it is likely that the use of teriparatide will be concentrated initially in patients with severe osteoporosis, with a treatment duration of probably 18 to 24 months, and with a transition to an antiresorptive agent at the end of that time. In clinical trials the drug has been well tolerated, producing only mild transient hypercalcemia, a moderate increase in urine calcium, and an unexplained mild increase in serum uric acid-all without symptoms. When given in large doses to rats through most of their lifespans, teriparatide significantly increases the risk of osteosarcoma. There have been no such reports in humans in clinical trials performed to date, nor is osteosarcoma a problem encountered in primary hyperparathyroidism. Rare outcomes of drug therapies are almost impossible to predict in advance. However, given the much shorter relative duration of dosing when teriparatide is used for the treatment of human osteoporosis, together with a basal incidence of osteosarcoma much lower in humans than in rats, it seems relatively unlikely that osteosarcoma will be a problem in the clinical use of teriparatide to treat human osteoporosis.

\section{ANATOMICAL RESTORATION FOLLOWING VERTEBRAL FRACTURE}

Spine compression fractures are one of the few skeletal fractures that, to date, have not been routinely treated by attempts to stabilize the fracture and to restore normal anatomical alignment and structure. Yet it is generally recognized that progressive deformity leading to kyphosis causes both chronic back pain and reduced functional capacity. Hence there has been considerable interest generated by two 
new minimally invasive procedures, kyphoplasty and vertebroplasty, which have as their purpose either the stabilization of a vertebral fracture, or the restoration of some of the loss of vertebral height. ${ }^{27}$ In both, a large bore needle is inserted from the back through the vertebral pedicle into the center of the vertebral body and bone cement is inserted. With kyphoplasty, balloon tamponade precedes the injection of cement to push apart the vertebral endplates, restoring some of the lost anterior height and creating a cavity. The cavity is subsequently filled with bone cement.

Results to date indicate immediate pain reduction with both procedures, as well as substantial ongoing functional improvement. Nevertheless, more time will be required to evaluate whether the introduction of a rigid material into the cancellous bone of the vertebral bodies alters the mechanical competence of the total spine, possibly leading to accelerated damage in adjacent vertebrae. Furthermore, studies assessing the long-term benefit of restoring weight-bearing alignment have yet to be performed. (However, it should be noted that such efficacy studies have essentially never been done for fracture-setting procedures for any other bone in the skeleton).

Closely related to these procedures is a type of vertebral fracture which, until very recently, had gone unrecognized, that is an unstable fracture or, effectively, a non-union. ${ }^{28}$ In these fractures, which seem to be concentrated near the thoracolumbar junction, 29 the vertebral body exhibits substantial compression when $\mathrm{x}$-rayed under vertical loading, but seems to be restored to normal shape when x-rays are taken with the back in hyperextension. This is not, of course, because the bone is flexible, but because the fracture opens and closes depending upon the forces imposed upon the spine at the time the x-ray is taken. These fractures generally cause continuing pain, often disabling, and can best be recognized by comparing lateral spine films taken first in the upright position, then in the supine position, particularly with a bolster under the back so as to exaggerate the lumbar lordosis. It has been McKiernan's experience ${ }^{29}$ that these fractures stabilize very nicely with low-pressure vertebroplasty, performed with the back in hyperextension. (This position opens the fracture and thereby creates a cavity for the bone cement to fill, i.e., using gravity and posture to do the job that, with kyphoplasty, is done with balloon tamponade).

\section{REMODELING AND FRAGILITY}

With the exception of teriparatide, all other bone-active agents work by suppressing remodeling activation and osteoclastic bone resorption. They thereby slow or arrest bone loss and, because of partial closure of the remodeling space (the amount of bone "out of commission" because it is currently being remodeled), they commonly produce a positive remodeling transient amounting, on average, to slightly less than $5 \%$ at one year of therapy. ${ }^{22}$ (The effect is called a "transient" because it occurs once on initiation of therapy, and an opposite change occurs when therapy is withdrawn.
In individuals with very high remodeling activity levels, this transient can amount to as much as a $30 \%$ gain in measurable bone mineral.)

Additionally, because resorption is suppressed more than formation, the anti-resorptive agents lead to a positive tissue level bone balance, i.e., to true bone gain. It has been argued that some of the increase in bone mineral density following anti-resorptives is as a result of secondary mineralization (the slow completion of mineralization after osteoblast activity has ceased). This is certainly correct, but the effect seen with the anti-resorptives is larger than can be accounted for on that ground alone, and substantially more prolonged. Long-term data with alendronate indicate that there is continuing bone gain, even if relatively slow, for as long as 7 to 8 years after starting treatment. ${ }^{30}$

Such gain in bone mass fits neatly into the bone mass paradigm, at least qualitatively. Implicitly the field has been operating under the presumption that the fragility was due primarily to decreased bone mass, and that any agent that stopped or reversed bone loss would decrease skeletal fragility, at least relative to the non-treated state. However, very recently Cummings published an analysis of the fracture risk reduction reported for the various anti-resorptive agents, and has calculated that less than half the observed improvement can be attributed to effects on bone mass. ${ }^{31}$ In fact, for most of the agents, $60 \%$ to $70 \%$ of the fracture risk reduction appears to have some basis other than bone mass protection or gain.

Sarkar et al., for example, showed, for the raloxifene-treated patients in the Multiple Outcomes of Raloxifene Evaluation (MORE) study, that simply being on raloxifene reduced fracture risk by about $40 \%$, even when bone mineral density did not change at all. ${ }^{32}$ In fact, the gradients of fracture risk on bone mass density (BMD) for the placebo and the raloxifene-treated patients were substantially different. This effect is illustrated in figure 3 for a hypothetical study of a placebo and any one of the anti-resorptive agents. As can be seen, while fracture risk varies inversely with BMD for both

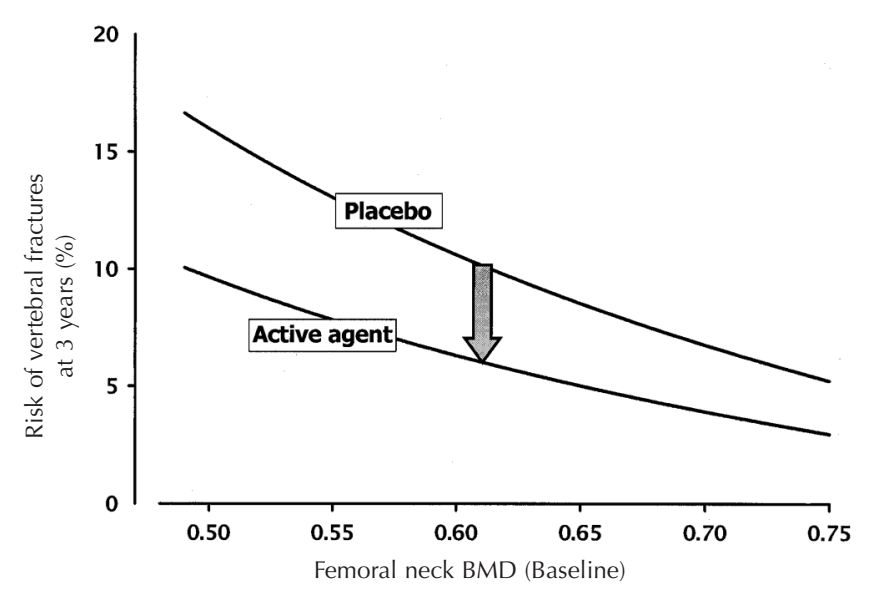

Figure 3. Gradients of fracture risk on BMD $\left(\mathrm{g} / \mathrm{cm}^{2}\right)$ for patients with osteoporosis and for patients treated with anti-resorptive agents, based on data developed for raloxifene. 29

Copyright Robert P. Heaney, 1994. Used with permission. 
placebo and anti-resorptive agent, for any given bone density, the fracture risk in the treated group is substantially lower.

Secondary analysis of some of the reported trials ${ }^{33}$ has indicated that the fracture risk reduction begins as early as three months, and most of the fracture benefit has been achieved by the end of the first year of treatment, well before the ultimate bone mass effect could have expressed itself.

The only known bony change that follows this time course is the suppression of bone remodeling, which occurs almost immediately on institution of anti-resorptive therapy. Remodeling weakens trabecular bone because remodeling loci, temporarily containing less bone, are inherently weaker than adjacent bone, and also because they concentrate and focus mechanical stresses experienced by the structure during routine loading. The probable importance of remodeling in fracture risk is explicitly supported by the observation from the European Patent Information and Documentation Systems (EPIDOS) study that basal remodeling rate predicts fracture. 34

The results of the two large controlled trials using only calcium and vitamin $\mathrm{D}$ also point to an effect of remodeling suppression. In these trials, in which reductions in non-vertebral fracture risk of $40 \%$ to $55 \%$ were reported, the effect began essentially immediately on institution of nutritional supplementation. ${ }^{17,18} \mathrm{~A}$ feature common to all of these situations is elevated basal remodeling, suppressed in one case by the anti-resorptive agents, and in the other by high-dose calcium and vitamin $\mathrm{D}$.

In light of these observations, it now must be seriously considered whether much of the baseline fragility of osteoporosis is due not only to decreased bone mass (which certainly aggravates the problem), but to high remodeling rates that we now recognize to be characteristic of many patients with this disorder. ${ }^{34,35}$ It follows automatically, therefore, that antiresorptives would reduce fragility in direct proportion to their effect on remodeling, and this appears to be precisely the case. Methods for assessing remodeling rate prospectively are improving rapidly, 35 but still lag behind the technology of bone mass measurement by perhaps 15 to 20 years. The growing recognition of the importance of remodeling should spur the development of needed improvements in this regard.

However, even at this stage of the evolution of technology, it seems safe to recommend use of remodeling biomarkers as a means of monitoring both compliance with anti-resorptive therapy and progress of treatment. If one has observed a substantial reduction in biomarker level, 3 to 6 months after starting an anti-resorptive agent, it seems reasonably safe to assure the patient that the drug is producing its desired effect, i.e., a reduction in fracture risk. BMD monitoring remains useful, but absence of BMD change by itself does not indicate treatment failure, especially if there is evidence of remodeling suppression.

\section{CONCLUSION}

The field of osteoporosis and its treatment continues to evolve, and more treatment agents, diagnostic methods and means of evaluating response are being introduced each year. Experience with the disorder forces the physician to recognize also that osteoporosis is a multifaceted problem and that an adequate approach in all of patients will require attention to many factors. We have better medical ammunition every day, but no single magic bullet.

\section{REFERENCES}

1. Consensus Development Conference: Prophylaxis and treatment of osteoporosis. Am J Med 1991;90:107-110.

2. Kanis JA, Melton LJ III, Christiansen C, Johnston CC, Khaltaev $\mathrm{N}$. The diagnosis of osteoporosis. J Bone Miner Res 1994;9:1137-1141.

3. Hui SL, Slemenda CW, Johnston CC. Age and bone mass as predictors of fracture in a prospective study. J Clin Invest 1988;81:1804-1809.

4. Lauritzen JB, Petersen MM, Lund B. Effect of external hip protectors on hip fracture. Lancet 1993;341:11-13.

5. Kannus P, Parkkari J, Niemi S, Pasanen M, Palvanen M, Jarvinen M, Vuori I. Prevention of hip fracture in elderly people with use of a hip protector. N Engl J Med 2000;343:1506-1513.

6. Cummings SR, Nevitt MC. A Hypothesis: The causes of hip fractures. J Gerontol 1989;44:M107-M111.

7. Moynihan R, Heath I, Henry D. Selling sickness: The pharmaceutical industry and disease mongering. BMJ 2002;324:886891.

8. Moynihan R, Smith R. Too much medicine. BMJ 2002;324:859860.

9. Smith R. In search of "non-disease". BMJ 2002;324:883-885.

10. Melton LJ III. Adverse outcomes of osteoporotic fractures in the general population. J Bone Miner Res 2003. In press.

11. Siris ES, Miller PD, Barrett-Connor E, Faulkner KG, Wehren LE, Abbott TA, Berger ML, Santora AC, Sherwood LM. Identification and fracture outcomes of undiagnosed low bone mineral density in postmenopausal women. JAMA 2001;286:2815-2822.

12. Gardner MJ, Flik KR, Mooar P, Lane JM. Improvement in the undertreatment of osteoporosis following hip fracture. J Bone Joint Surg 2002;84A:1342-1348.

13. Reid IR, Brown JP, Burckhardt P, Horowitz Z, Richardson P, Trechsel U, Widmer A, Devogelaer JP, Kaufman JM, Jaeger P, Body JJ, Meunier PJ. Intravenous zoledronic acid in postmenopausal women with low bone mineral density. N Engl J Med 2002;346:653-661.

14. Dietary Reference Intakes for Calcium, Magnesium, Phosphorus, Vitamin D, and Fluoride. Food and Nutrition Board, Institute of Medicine. National Academy Press, Washington, DC, 1997.

15. Heaney RP, Nordin BEC. Calcium effects on phosphorus absorption: implications for the prevention and co-therapy of osteoporosis. J Am Coll Nutr 2002;21:239-244.

16. Heaney RP, Davies KM, Chen TC, Holick MF, Barger-Lux MJ. Human serum 25-hydroxy-cholecalciferol response to extended oral dosing with cholecalciferol. Am J Clin Nutr 2003;77:204-210.

17. Chapuy MC, Arlot ME, Duboeuf F, Brun J, Crouzet B, Arnaud $\mathrm{S}$, Delmas PD, Meunier PJ. Vitamin D3 and calcium to prevent hip fractures in elderly women. N Engl J Med 1992;327:1637-1642.

18. Dawson-Hughes, B., Dallal, G.E., Krall, E.A., Sadowski, L., Sahyoun, N., Tannenbaum, S. A controlled trial of the effect of calcium supplementation on bone density in postmenopausal women. N Engl J Med 1990;323:878-883. 
19. Delmi M, Rapin CH, Bengoa JM, Delmas PD, Vasey H, Bonjour JP. Dietary supplementation in elderly patients with fractured neck of the femur. Lancet 1990;335:1013-1016.

20. Schürch MA, Rizzoli R, Slosman D, Vadas L, Vergnaud P, Bonjour JP. Protein supplements increase serum insulin-like growth factor-I levels and attenuate proximal femur bone loss in patients with recent hip fracture. Ann Intern Med 1998; 128:801-809.

21. Dawson-Hughes B, Harris SS. Calcium intake influences the association of protein intake with rates of bone loss in elderly men and women. Am J Clin Nutr 2002;75:773-779.

22. Heaney RP, Yates AJ, Santora AC II. Bisphosphonate effects and the bone remodeling transient. J Bone Miner Res 1997;12:1143-1151.

23. Saag KG, Emkey R, Schnitzer TJ, Brown JP, Hawkins F, Goemaere S, Thamsborg G, Liberman UA, Delmas PD, Malice MP, Czachur M, Daifotis AG. Alendronate for the prevention and treatment of glucocorticoid-induced osteoporosis. N Engl J Med 1998;339:292-299.

24. Lane NE, Sanchez S, Modin GW, Genant HK, Pierini E, Arnaud CD. Bone mass continues to increase at the hip after parathyroid hormone treatment is discontinued in glucocorticoid-induced osteoporosis: Results of a randomized controlled clinical trial. J Bone Miner Res 2000;15:944-951.

25. Neer RM, Arnaud CD, Zanchetta JR, Prince R, Gaich GA, Reginster JY, Hodsman AB, Eriksen EF, Ish-Shalom S, Genant HK, Wang O, Mitlak BH. Effect of parathyroid hormone (1-34) on fractures and bone mineral density in postmenopausal women with osteoporosis. N Engl J Med 2001;344:1434-1441.

26. Arnaud CD, Roe EB, Sanchez MS, Bacchetti P, Black DM, Cann CE. Two years of parathyroid hormone 1-34 and estrogen produce dramatic bone density increases in postmenopausal osteoporotic women that dissipate only slightly during a third year of treatment with estrogen alone: Results from a placebo-controlled randomized trial. Bone 2001;28:S277.

27. Watts NB, Harris ST, Genant HK. Treatment of painful osteoporotic vertebral fractures with percutaneous vertebroplasty or kyphoplasty. Osteoporos Int 2001;12:429-437.

28. Faciszewski T, McKiernan F. Calling all vertebral fractures. Classification of vertebral compression fractures: a consensus for comparison of treatment and outcome. J Bone Miner Res 2002;17:185-191.

29. McKiernan F, Jensen R. The dynamic mobility of vertebral compression fractures. J Bone Miner Res 2003;18(1):24-29.

30. Tonino RP, Meunier PJ, Emkey R, Rodriguez-Portales JA, Menkes CJ, Wasnich RD, Bone HG, Santora AC, Wu M, Desai R, Ross PD. Skeletal benefits of alendronate: 7-year treatment of postmenopausal osteoporotic women. J Clin Endocrinol Metab 2001;86:1835-1836.

31. Cummings SR, Karpf DB, Harris F, et al. Improvement in spine bone density and reduction in risk of vertebral fractures during treatment with antiresorptive drugs. Am J Med 2002;112:281-289.

32. Sarkar S, Mitlak BH, Wong M, Stock JL, Black DM, Harper KD. Relationships between bone mineral density and incident vertebral fracture risk with raloxifene therapy. J Bone Miner Res 2002;17:1-10.

33. Felsenberg D, Hanley DA, Johnson TD, Manhart MD, Genant HK. Risedronate significantly reduces moderate and severe vertebral fractures by $70 \%$ in one year. J Bone Miner Res 2002;:Suppl 1:S207.

34. Garnero P, Hausherr E, Chapuy MC, et al. Markers of bone resorption predict hip fracture in elderly women: the EPIDOS prospective study. J Bone Miner Res 1996;11:1531-1538.
35. Looker AC, Bauer DC, Chesnut CH III, Gundberg CM, Hochberg MC, Klee G, Kleerekoper M, Watts NB, Bell NH. Clinical use of biochemical markers of bone remodeling: current status and future directions. Osteoporos Int 2000;11:467480 . 